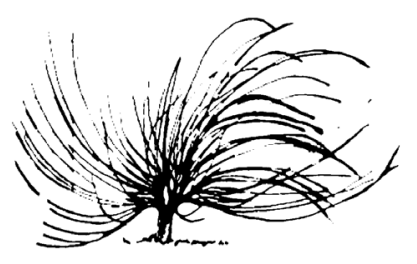

\title{
El impacto de la globalización en la educación superior del siglo XXI. Cambios en el imaginario social de las comunidades educativas
}

\author{
Ignacio Acosta Ballestero ${ }^{1}$ \\ Universidad Nacional \\ Costa Rica \\ iacostaballestero86@gmail.com
}

\begin{abstract}
Resumen
En este ensayo, se realiza un balance reflexivo sobre los cambios provocados por la globalización, en su etapa neoliberal, en el imaginario social de las comunidades educativas en la educación superior durante el siglo XXI. Para lograrlo, se realiza un acercamiento contextual discursivo sobre los efectos de la globalización generados a nivel nacional e internacional, para posteriormente profundizar sobre el cambio en las dinámicas y las posibilidades para tomar consciencia crítica en relación con las implicaciones de estos movimientos. Además, en este análisis se discute sobre la necesidad de tomar en cuenta a las nuevas generaciones de estudiantes con quienes vamos a trabajar y poder observar estos escenarios, como espacio para la transformación de los procesos de enseñanza y aprendizaje.
\end{abstract}

Palabras clave: Globalización, neoliberalismo, imaginario social, comunidades educativas, educación Superior, enseñanza y aprendizaje.

http://dx.doi.org/10.15359/rep.esp-21.6

1 Licenciado en La Enseñanza de los Estudios Sociales y Educación Cívica de la Universidad Nacional de Costa Rica, Bachiller en la Enseñanza de los Estudios Sociales y Educación Cívica de la Universidad Nacional de Costa Rica, Bachiller en Historia de la Universidad Nacional de Costa Rica.

ORCID ID. https://orcid.org/0000-0002-3759-2826 


\begin{abstract}
In this paper, a reflexive assessment of the shifts in the social imaginary of the educational communities in higher education of the 21st century generated by the process of globalization in its neoliberal juncture is made. To achieve this endeavour, a contextual discourse analysis about the effects of globalization on international and domestic levels has been carried out in order to deepen on the dynamics and the opportunities to raise awareness of the implications of this type of manifestations. In addition, this analysis aims to take into account the needs of the students of the future generations and works to open the possible scenarios as spaces to transform the teaching and learning process.
\end{abstract}

Keywords: globalization, neoliberalism, social imaginary, educational communities, higher education, teaching and learning

\title{
Introducción
}

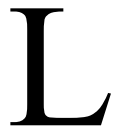

a educación superior ha atravesado procesos de cambios y transformaciones propios de su razón de ser, generados por dinámicas marcadas a través del análisis de los espacios contextuales en los que se desarrollan y por su implementación como parte de la búsqueda de respuestas a las necesidades imperantes en el entorno nacional e internacional. Estas situaciones vigentes, exigen compromiso para que dentro de este ensayo se tome en cuenta el impacto producido por la globalización en el escenario de finales del siglo XX y de las primeras décadas del siglo XXI (Gurdián, 2010).

En este proceso se puede observar un elaborado tejido de juegos de poder, influencias generadas por grupos hegemónicos, colectivos conscientes de las necesidades propias de una institución en específico, actores especialistas en esta área, autoridades y el estudiantado mismo. Este cúmulo de personajes generan espacios desde donde surgen interrogantes que dificultan el reconocimiento de las intencionalidades por las luchas y los objetivos de cada una de las agrupaciones, entendidos como posibilidades para la transformación en los espacios de educación superior o la reproducción de dinámicas de imposición que benefician de forma mayoritaria a las estructuras de poder comentadas. 
Por ende, como parte de los procesos que han venido ocurriendo en las últimas décadas de finales del siglo XX y principios del XXI, en esta reflexión se vuelve trascendental tomar en cuenta los proyectos de los distintos conglomerados que participan en dicho contexto, para reconocer su influencia y los niveles de incidencia en las comunidades educativas y en el imaginario social.

Los cuestionamientos de estas situaciones expuestas se relacionan con las posturas de análisis planteadas en este ensayo. Como referentes, se utiliza una combinación entre la teoría crítica y la pedagogía crítica para confrontar las estrategias de control, el interés o desinterés de personajes claves en relación con la educación superior y, la implementación de cambios en los niveles educativos desde donde se desprende también la cantidad de recursos destinados a los programas de innovación y mejora (Streck et al., 2015)

En este entramado, se encuentran argumentos fundamentales del presente enfoque. Los razonamientos giran entre los caminos de quienes miran el contexto marcado por la incidencia de nuevas etapas de globalización como un espacio de oportunidades y, comunidades afiliadas al cumplimiento de manera rutinaria y al pie de la letra, de las políticas neoliberales impulsadas desde organizaciones como el Banco Mundial y el Fondo Monetario Internacional.

En el ámbito nacional, la discusión mantiene la misma línea discursiva: los cambios propuestos desde los organismos de gestión universitaria, el mejoramiento de los procesos educativos, la reformulación de las carreras, la acreditación y la discusión sobre la construcción de conocimiento, encuentran parangón con respecto a la reflexión crítica sobre su implementación como escenario de desafíos, pero también como abanico de posibilidades.

Este escenario brinda la posibilidad para reconocer las dinámicas de la globalización en la etapa neoliberal y sus modificaciones en el imaginario social de las comunidades educativas de nivel superior. Estos cambios se reflejan en la interacción cotidiana dentro de las aulas, la vinculación con el estudiantado, la producción de conocimiento o su construcción crítica, permitiendo así, considerar cómo los espacios abiertos de reflexión, posibilitan construir puentes para tomar consciencia sobre la incidencia social, económica y política de la globalización en la educación superior y las posibilidades para embarcarse hacia mares de transformación educativa, de la mano con el trabajo desde las costas 
cargadas de posibilidades, de la esperanza y el compromiso constante de comprender el contexto y las generaciones de estudiantes con quienes se intercambiarán espacios para la construcción de conocimientos.

\section{Acercamiento desde el contexto internacional}

Para profundizar sobre la incidencia de la globalización, enmarcada en la etapa neoliberal ${ }^{2}$, en relación con la educación superior del siglo XXI y el imaginario social de sus comunidades educativas, se hace necesario plantear una serie de mojones que permitirán reconocer los cambios producidos por esta serie de situaciones dentro de los escenarios educativos y sociales donde participan diferentes profesionales. Por ende, se plantea como punto de partida exponer algunas de las decisiones tomadas a nivel internacional en relación con las políticas en esta área y sus efectos para las comunidades educativas.

Al partir de este escenario, se reconoce que Costa Rica se ha visto marcado por transformaciones políticas de corte macroeconómico. Se inicia con sus retratos, con el fin de comprender cómo su aplicación ha generado modificaciones en la vivencia de los conjuntos sociales y la interacción de las personas dentro de espacios como la educación superior. Estos cambios pueden ser observados desde lo tangible y elaborado, hasta llegar a los aspectos intangibles. De esa manera, por medio del análisis crítico se comprenden los niveles de influencia, e intencionalidades de los diferentes grupos que pretenden implementar dichos cambios en los escenarios educativos.

La reflexión planteada parte de las últimas décadas del siglo XX, donde las políticas de Estado se ven marcadas por la participación, cada vez mayor, de organismos como el Banco Mundial y el Fondo Monetario Internacional. La situación económica y de dependencia imperante en muchos de los territorios de América Latina y el Caribe, desde el periodo colonial, ha provocado el desarrollo de patrones que conducen de una manera u otra, a entornos en donde la participación de los

2 En este ensayo, se plantea el uso del término globalización en la etapa neoliberal, desde el acercamiento que vislumbra escenarios compartidos, donde las políticas de Estado y mercado a escala mundial internacional, se articulan de manera conjunta, sin borrar los espacios de incidencia de uno y otro, pero reconociendo las formas de dominio político en los casos particulares y globales en la manera de expresarlo sobre los conjuntos sociales a partir del control de capital. Para esto, se expone cómo la globalización, desde el punto de vista histórico, no corresponde a un proceso nuevo, sino como un cúmulo de situaciones que toman pertinencia desde nuestro enfoque a partir de los cambios producidos a nivel económico mundial desde la década de 1970, en donde cobran relevancia las políticas de corte neoliberal. 
organismos mencionados, corresponde al rescate de las economías, a cambio de modificaciones sustanciales en la estructura económica-política de los países en cuestión. Esta situación provoca como consecuencia la disminución en la forma como se distribuyen los presupuestos destinados a solventar necesidades de la población.

En este proceso, se pasa a reflejar el cambio producido a partir de la caída de modelos de Estado. En la etapa previa, estuvieron caracterizados por mayor participación e inversión en políticas de corte social, situación que, para algunas escuelas económicas causó el deterioro de las economías de los países que las implementaron. Luego, se produjo la transición hacia nuevos modelos de gestión estatal. En ellos existe mayor contención hacia este tipo de programas sociales, en la búsqueda de mayor eficiencia desde políticas que cambien la estructura y procuren mantener la estabilidad para sacar a flote las economías dependientes. Lo mencionado anteriormente, ha perpetuado la hegemonía histórica de los ejes de poder y control (Sánchez y Zaldívar, 2015).

La conjugación de estas situaciones, sumado a siglos de exclusión y dominación han generado y aumentado la desigualdad, mostrada en niveles relacionados con el acceso a la educación, las posibilidades de ascenso social, la mejora en las condiciones de vida, la atención hacia las necesidades básicas, la disminución de los índices de pobreza y el analfabetismo. Al reducirse los fondos destinados a atender dichas necesidades, se produce por efecto el aumento de la desigualdad en los grupos poblacionales con mayores carencias (Ferrer, 2013).

Con estos antecedentes, las discusiones giran en torno a los fines que pretende la educación, desde puntos de vista que toman en cuenta los procesos educativos como un gasto hasta aquellos que la vislumbran como una inversión. En estos ámbitos, se comprende el proceso educativo como una serie de pautas determinadas por las demandas del mercado, así como por intereses de agrupaciones cuyo enfoque se basa en aproximaciones con mayores grados de criticidad y consciencia en relación con las dinámicas del contexto actual. Esta discusión no es nueva, se puede encontrar en la lucha teórica discursiva y práctica entre los defensores de la pedagogía crítica y la pedagogía tradicional ${ }^{3}$. No

3 Según (Escárcega, 2014, p. 28) "la Teoría del Capital Humano coloca a la educación en una posición en donde es vista como una inversión por parte del Estado para generar réditos a corto y largo plazo. Por ende, es necesario ofertar, vender, consumirla y si fuera el caso desecharla". Se pretende, bajo esta premisa, preparar a las personas que esa figura y modelo de Estado 
obstante, desde las políticas neoliberales expandidas por medio de la globalización, la lupa se enfoca en las transformaciones que las medidas provocan en sus nichos de acción.

En la presente coyuntura histórica, a partir de los eventos ocurridos en la década de 1970, con la compresión económica, hasta las primeras décadas del siglo XXI, la visión de las políticas neoliberales ha planteado la reducción del gasto social. Esto sucede debido a los puntos de vista de quienes impulsaron estas políticas, en donde la apertura de programas de corte social y educativo, destinados a poblaciones en necesidad, representa desembolsos directos para el erario público. Esto se convierte en el principal foco de presión presupuestario y el generador de inestabilidad económica para los gobiernos de turno (Pascual y Ghiotto, 2010).

De manera paralela, las transformaciones en la figura del Estado, enfiladas desde los organismos internacionales mencionados, y el plan económico neoliberal, producen nuevos enfoques en la forma de gestionar los presupuestos. Según Apple (2001), las nuevas estrategias gubernamentales están pensadas para que desde los centros educativos se enseñe lo necesario, manteniendo bajo control de la colonialidad hegemónica, los conocimientos y mecanismos establecidos por las estructuras de poder, para controlar los saberes de los pueblos de acuerdo con las necesidades del capital (Lander, 2000).

Estas dinámicas incitan al establecimiento de nuevos parámetros para destinar los fondos, desde la proyección discursiva sobre el control del gasto y eficiencia en las maneras en las cuales se utilizan los recursos. Esto conlleva a que en las disertaciones sobre la implementación de las políticas educativas se haga común optar por la inversión en innovación, desde los ámbitos estructurales visibles y cuantificables, la utilización de la tecnología, la informática, robótica, el manejo de paquetes de software y el hardware vinculado con las necesidades del contexto del mercado.

En ese sentido, para su desarrollo se fomentan reformas educativas de carácter curricular, con incidencia en las normativas institucionales, así como en la cotidianidad de los procesos de enseñanza y aprendizaje (Blanco et al., 2017). Por lo que este proceso educativo, 
instrumentaliza las etapas en relación con las posibilidades de producción y la función de respuesta a las demandas del mercado. Es decir, a la preparación de un estudiantado apto para ese entorno, con los apoyos educativos prácticos y necesarios para cumplir con las funciones que los puestos le demandarán.

Ante la influencia de estos factores, las posibilidades para el desarrollo adecuado de una educación integral se van dejando de lado. Esto sucede debido a la influencia de elementos de corte presupuestario mencionado, la competencia generada por el mercado, relacionada con la preparación de ese tipo de estudiantes, falta de tiempo, y a la insuficiente capacidad, o disposición, para encontrar sentido práctico a procesos educativos que generen saberes en las personas que les permita reconocer la manipulación de las fuerzas hegemónicas (Huerta, 2005). De acuerdo con lo analizado y expuesto anteriormente, se aprueba decir que las políticas neoliberales han aprovechado los canales abiertos por la globalización, para reproducir la concepción de producción mecánica y éxitos a nivel académico con su consecuente respuesta en la balanza económica.

Con más frecuencia se reproduce el uso de la palabra "calidad", además de la "estandarización de los conocimientos, el individualismo y la competencia" ", como respuesta a la reproducción de los saberes necesarios para ese contexto determinado por el mercado. En ese caso, desde esta visión, el contenido ideológico neoliberal está presente y altera las subjetividades de las personas en su cotidianidad, pues con la implementación de las políticas, los programas sociales y la educación son ofrecidos como servicios, no como derechos fundamentales para las personas. El éxito de estos mismos radica en que profundizan de tal manera, en el imaginario de los grupos sociales, que son los últimos quienes defienden la necesidad de gestar cambios en aras de vincularse con los procesos económicos imperantes.

En medio de este imaginario social, las líneas maestras van tejiendo procesos de desarraigo y ruptura en las nuevas generaciones. Ellos se vinculan con componentes culturales presentes desde siglos

4 Se habla de individualismo inmerso en las nuevas dinámicas de la post modernidad. Esta situación se refleja en la manera en la cual se producen las relaciones sociales dentro y fuera de las instituciones educativas. Con mayor claridad, se notan los esfuerzos por derribar cualquier tipo de oposición. De esa manera, domina solo una forma de pensar, afianzada y reforzada por las propuestas de un imaginario liberal (Torres, 2001). 
pasados. La intención reflejada, pretende que este proceso no se origine con demasiada rapidez y sea disruptivo. Como resultado de dicha situación, las relaciones hegemónicas presentes en estas situaciones, desarrollan sociedades con falta de conciencia y sentido de pertenencia hacia las comunidades en las que habitan y con las que interactúan.

Por ende, el individualismo fomentado por la globalización, repercute en el desinterés por la cooperación y actitudes solidarias dentro y fuera de la institución educativa. De acuerdo con Castells (2001), esto provoca comunidades desarraigadas, en donde solo interesa el individuo, sus posesiones. Esta situación produce resultados nefastos en situaciones que busquen o generen beneficios para el colectivo. Es estos casos, es preferible el bienestar de un individuo que la prosperidad del conjunto.

De la misma manera, la falta de sitios de esparcimiento, la sensación de inseguridad y el vínculo casi nulo con la comunidad arrastran a muchos jóvenes a consumirse dentro de las casas. Según Torres (2001), entonces, se pierden las herramientas de socialización en espacios públicos y políticos cuando "[1]a moderna sociedad de economía neoliberal encarna la victoria de la vertiente económica, de la victoria del animal laborans, convirtiendo el mundo en el espacio del homo económicus" (p. 25).

Los pasos retratados permiten observar los patrones relacionados con las condiciones imperantes. Ellas han cambiado la percepción de las comunidades, desde las dinámicas de conjunto hacia la primacía de la competencia individualizada. Además, para el cumplimiento de los planes propuestos, desde las políticas neoliberales, se ha implantado, de forma procesual, el uso de vocabularios que exhortan a la búsqueda de mejores espacios para la población en general, se normaliza su uso, la defensa de los objetivos, pero no la comprensión crítica de las implicaciones que su utilización, dentro los espacios sociales, educativos, culturales y, económicos-estatales pueda producir.

Dicha situación ocurre debido a que en los discursos se incluye, de forma perceptible o imperceptible, la pretensión de defensa de las oportunidades, la gestión efectiva por medio de las buenas prácticas, la idoneidad de los procesos, la estandarización de criterios, la vigilancia en cada una de las etapas para así asegurar el cumplimiento de las funciones. Todos estos vocablos tienen su nicho desde las posturas económicas, se dejan de lado los aspectos humanos, integrales y las diferencias contextuales del proceso educativo para poder implementarlas. 
Esto nos lleva, como colectivo, a tomar consciencia sobre el potencial relacionado con la estrategia sistemática del uso del lenguaje y la gramática del poder con las maneras como los discursos calan hasta lo más profundo de la médula de los tejidos sociales y los procesos de enseñanza y aprendizaje. Bajo esas condiciones, la misma epistemología se encuentra alineada con los procesos económicos y políticos enfilados desde las ideas y puestas en práctica del neoliberalismo. Esto conduce a la inserción de enunciados, teorías, imágenes vinculadas con las lógicas de producción, así como la transformación del imaginario social (Solano, 2018).

De acuerdo con lo expuesto, las condiciones imperantes hacen que la preparación del profesorado y del estudiantado dentro de la educación superior, atraviese una serie de complicaciones, en cuanto a parámetros marcados por las posibilidades de acceso, destino presupuestario para el cumplimiento de funciones relacionadas con la investigación y extensión, la necesidad para revalorizar los objetivos de algunas carreras, cerrar carreras con baja demanda, esquemas de contratación que producen inestabilidad en el profesorado, precarización de las instalaciones, dependencia de las normativas y prácticamente manos atadas para tomar decisiones de gestión más alejadas de la estrategia sistemática de mercado.

Como se puede observar, este cúmulo de situaciones expuestas se caracteriza por la participación de varios grupos. Ellos intentan plasmar sus intenciones por medio de la implementación de políticas a nivel estructural y de esfuerzos para modificar de forma sistemática la comprensión del presente entorno, incluida la cotidianidad. Esto produce cambios en la población estudiantil, en el profesorado, en las personas encargadas de la administración de cada una de las unidades académicas y el sistema educativo de educación superior.

En ese sentido, de acuerdo con lo revisado, la implantación de las políticas neoliberales corresponde a la serie de estrategias utilizadas, mayoritariamente por los grupos hegemónicos, para establecer una cadena de condiciones en los grupos poblacionales y la globalización se convierte en el medio para transmitir esas estrategias. Es menester continuar abriendo posibilidades para reflexionar, repensar y discutir sobre las implicaciones producidas por estos fenómenos, en la medida que permiten comprender la necesidad por la preparación constante dentro de los espacios educativos. 


\section{Contextualización a nivel nacional}

Con este escenario plasmado, se procede a profundizar la discusión sobre los alcances causados en el actual contexto, inmerso en las condiciones propias del caso costarricense. Esas particularidades son las que definen la ruta de este trabajo, , así como la presentación de los desafíos y cambios producidos dentro de las comunidades educativas en la educación superior.

Así pues, se parte de las consideraciones expuestas por Núñez (1974) en relación con la universidad necesaria, los desafíos vinculados con cambios significativos en la estructura de la educación superior, la sociedad y la construcción del conocimiento. Esta situación conllevó construir espacios para la reflexión e interpretación de la realidad. Dichas aproximaciones se toman en cuenta al reconocer que las propuestas se encontraron afincadas en un momento histórico distinto, con diferentes condiciones, fuerzas políticas internas y externas, otras intencionalidades, así como la visión estatal con texturas singulares, pero que marcaron el camino para esfuerzos colectivos dentro de los ámbitos educativos universitarios.

Sin embargo, con estas líneas no se pretende caer en la trampa de vanagloriar otras épocas y reproducir una visión acrítica sobre tiempos pasados. Por esta razón, son esbozadas en este espacio, como punto de partida para las reflexiones relacionadas con la influencia de la globalización en la educación superior y en las comunidades educativas durante la coyuntura de los primeros años del siglo XXI.

Se retoma la deliberación sobre las consecuencias provocadas durante el periodo que dio fin a un modelo de estado gestor, para pasar a uno con visiones ligadas a la empresa y al mercado. Luego del periodo de crisis acontecido a principios de 1980 fue necesario renegociar la deuda. Esto produjo subsecuentes cambios en la estructura del país y el control sobre el presupuesto orquestado por influencia de las recomendaciones de organismos como el FMI y el Banco Mundial ${ }^{5}$. Esto

5 Se hace la acotación sobre los modelos de Estado, en el caso costarricense, como una muestra que refleja los cambios ocurridos en la Costa Rica de finales del siglo XX y principios del siglo XXI. No obstante, no se debe olvidar que, en muchos casos, los procesos educativos en Costa Rica, siguieron patrones importados de otros lugares, como Chile, España y Estados Unidos. Las reformas impulsadas estuvieron marcadas por la preparación de estudiantes que cumplieran funciones específicas y necesarias para la implantación de los modelos de desarrollo instaurados en el país. 
muestra el cúmulo de situaciones imperantes a partir de las últimas décadas del siglo XX y los desafíos constantes del siglo XXI.

La mayor participación en la toma de decisiones por parte de estos organismos sobre los gobiernos nacionales llevó a transformar los procedimientos y las formas como se habían generado procesos históricos en las décadas anteriores. Esta alteración en la velocidad y los flujos de cambio conllevó la construcción de necesidades o la percepción de las mismas, vinculadas con la innovación educativa, comprendida por algunos ministros y gobernantes de la época, como un recetario cuyos esfuerzos por cumplirlo a cabalidad, se enfocaron en el componente tecnológico. Es decir, la innovación partió de la preparación en informática, la adquisición de computadores, paquetes y cambios en la estructura curricular para sostener estos nuevos enfoques en el escenario educativo.

Para cumplir con estas exigencias, las instituciones de educación superior también tuvieron que ajustarse a las nuevas dinámicas planteadas por el contexto internacional y de esa manera se promovieron una serie de pasos que involucraron la preparación de profesionales en las áreas específicas demandadas por los nuevos proyectos de carácter estatal.

Se confieren efectos de multicausalidad ante dichas situaciones comentadas, puesto que, por un lado, se encuentran características específicas imperantes en el entorno internacional y nacional, que causaron cambios a lo interno de las carreras y la función social de las universidades. Mientras, por la otra arista, se vislumbra el efecto provocado por las mismas modificaciones, cuando se toman en cuenta las posibles consecuencias del rezago, si la ciudadanía no se suma a los procesos de cambio enlazados a la globalización.

Las disposiciones causaron que el contexto nacional se encontrara delimitado por espacios desde donde se fueron preparando modificaciones a nivel curricular, cambios en la base de las mismas unidades académicas y en la evaluación de las carreras, de acuerdo con las necesidades del nuevo siglo y las demandas de la visión de mundo globalizado instaurada, que por cierto, es cada vez más exigente para con el perfil de los profesionales que se están preparando.

Esas circunstancias vuelven la palestra de manera indistinta hacia las poblaciones con quienes se participa en la construcción de conocimiento. De esta manera, se han producido cambios en las prácticas cotidianas, formas de comunicación, interacción e intercambio de 
saberes, la praxis pedagógica, dentro y fuera de los salones de clase. Además, toda la dilatación y la competencia constante planteada en estos nuevos entornos, provoca transformaciones significativas en comunidades tan desiguales como las costarricenses. Estas dinámicas de influencia externa, que toman sentido en el país por medio de políticas estatales con efecto sobre los espacios sociales y educativos, han requerido también la constante revisión crítica por parte de las autoridades universitarias, el profesorado y componentes de la población estudiantil a lo largo de los años en cuestión. Con estos acuerdos, se ha abierto la posibilidad para poder trabajar de la mano con la flexibilidad y, de esa manera adaptar los espacios de educación superior a las demandas del mundo globalizado, pensar en el futuro sin dejar de lado los efectos sobre las comunidades, los procesos educativos imperantes y las situaciones ocurridas en el país.

En los ámbitos educativos, algunas de las demandas estuvieron relacionadas con el uso de las TIC. Para ello, fue necesario la restructuración, preparación y apertura de carreras vinculadas con esos requerimientos, mismos que tienen rastro desde finales de la década de 1970. La apertura de dichas carreras permitió la relación con las tecnologías, conexión por medio de la red, investigación en temas encadenados a la computación e informática y la preparación de personas para la llegada de empresas transnacionales que se establecieron a finales del siglo XX y posteriormente, en las primeras décadas del siglo XXI.

Tomar en consideración las situaciones vigentes a lo interno del país posibilita detectar cómo el inicio de dichos pasos, desvinculados de la investigación, de índole contextualizado y de la identificación de características excluyentes, aumentan las disparidades dentro de las comunidades educativas. Algunos de los factores que dificultan y generan diferencias, magnificadas por efecto de la implementación de estos planes sin el debido seguimiento corresponden a: el rezago, desigualdad entre las posibilidades de las zonas urbanas con respecto a las rurales, extensión y proyección de los servicios, la velocidad de la red con respecto al acceso a las tecnologías, brechas notables entre grupos generacionales (Estado de la Nación, 2011).

Esto ha generado y aumentado la sensación de competencia dentro de los espacios educativos. La falta de posibilidades se ve incrementada por las disparidades en el acceso a los conocimientos básicos, 
propios del uso de las tecnologías, situación que se hace evidente cuando se comparan zonas del país, sobre todo aquellas localizadas cercanías de las fronteras. En lo más profundo de la construcción de un imaginario social, las circunstancias descritas provocan tensiones, enojo, indignación, conflictividad, frustración y separaciones entre grupos de estudiantes o generaciones, dentro de las comunidades de aprendizaje. ${ }^{6}$

Por ende, se considera que, con el establecimiento de políticas de innovación educativa, vinculadas con programas de informática, computación, software y hardware, se pueden observar cúmulos de intencionalidades. Por un lado, se encuentran las que aprovechan la globalización para intereses del mercado y los que intentan utilizarla de manera transformadora, temática que se ha venido desarrollando a lo largo de este trabajo. Entre las intencionalidades se presentan las correspondientes al corte industrial mecánico, de preparación de profesionales que cumplen con las necesidades técnicas del mercado, conectados en este caso, con la posterior llegada de empresas como Intel y Hewllet Packard (Escuela de Ciencias de la Computación e Informática, s. f.). Mientras tanto, otra de las aristas se puede encontrar en aquellas personas, cuya visión pretendió convertir los avances traídos por la globalización y los desafíos del mercado, como entornos de posibilidades, para plantear escenarios de trabajo y consolidación de investigaciones a partir de ellas.

Este transcurrir lleva las reflexiones generadas a ámbitos donde se toman en cuenta las visiones relacionadas con la educación y su enfoque práctico presente en la cotidianidad del proceso de enseñanza y aprendizaje. Desde dichas perspectivas, se moldea la posición sobre la educación como derecho y sus efectos concernientes a su relación con el acceso al conocimiento y la posición de los ejes de poder, la posibilidad para descolonizar el acceso a los saberes y la carrera constante por el acceso a los mismos.

En el caso costarricense, según (Gurdián, 2010), la ruta se encuentra marcada por las políticas enviadas desde el Banco Mundial, en las cuales, el discurso versa sobre la importancia histórica de los conocimientos y el acceso a ellos, para generar oportunidades de inclusión

6 Para efectos de este ensayo se expone cómo, sin el debido cuidado y reflexión crítica, la utilización las tecnologías de la información y comunicación, lejos de convertirse en motores para la socialización, pueden resultar en disruptores de comunidades y disparadores de diferencias sociales, económicas y políticas. 
o exclusión de distintos grupos poblacionales. No obstante, las posibilidades prácticas para encarar estos procesos de forma autónoma por parte del Estado se reducen y se entregan las capacidades de decisión, en materia correspondiente al trasfondo de los procesos educativos, a los intereses generados por grupos empresariales transnacionales, que de por sí controlan la rutas que lleva el proceso de globalización.

La identificación del marco en el cual se han venido desarrollando los procesos a nivel educativo en general y en la educación superior en específico, ha provocado que las discusiones giren en torno a la calidad de la educación, la estandarización y la acreditación. Estos criterios, reproducidos como parte de discursos en los espacios educativos superiores (como los organismos regentes de la educación en el país), calan en lo más profundo de dichos escenarios y comienzan a transformar las dinámicas dentro de las comunidades educativas, unidades académicas, instancias de educación superior y las necesidades de profesionales del mercado y la población.

Como resultado de la afiliación a este marco de referencia, comenzaron a notarse con mayor periodicidad los recortes presupuestarios, cambios en las universidades públicas, apertura de cada vez más centros privados de educación superior. No obstante, con respecto a las modificaciones en las instituciones públicas y la apertura de centros privados, el denominador común fue la falta de control o mecanismos para certificar la calidad de los procesos realizados. Para cumplir con estos puntos, se establecieron una serie de parámetros que las instituciones debían cumplir y organismos específicos de carácter estatal constatar. Es decir, la tarea atravesó por la burocratización y regulación normativa desde la cual se han pretendido estructurar los procesos de enseñanza y aprendizaje de forma homogénea (Rama, 2017).

Los recortes presupuestarios se han vuelto un indicador de las últimas gestiones gubernamentales. Esto se refleja en la cantidad de fondos reales destinados para proyectos, que sobrepasen la intervención estructural y de compra de equipo necesario para la innovación educativa. En ese sentido, la cantidad de fondos para la educación superior pública disminuye con cada gestión, eso atenúa las posibilidades para destinar dineros a becas, proyectos de extensión e investigación, lo cual genera que el sentido de la educación superior se lleve hacia una mecanización competitiva y exclusiva del acceso al conocimiento. 
Las situaciones analizadas permiten reconocer cambios en la manera como se percibe la cotidianidad y los desafíos que conllevan estas dinámicas para todo el proceso de enseñanza y aprendizaje. Estos espacios de reflexión y contraste permitieron generar aproximaciones y reconocer los desafíos de la práctica educativa, de observación, socialización y acción en las instituciones de educación superior. De la misma manera, se posibilitó vislumbrar desde diferentes aristas, la influencia producida por la globalización, en las relaciones socioeducativas de la población educativa (docentes y estudiantes), encargados del proceso a nivel de las universidades y en la esfera país.

En relación con la ruta expuesta en los párrafos anteriores, se toma en cuenta la incidencia de la globalización en las prácticas cotidianas llevadas a cabo dentro de la educación superior. Por ende, se partió de intentar comprender este fenómeno, no como un único camino, sino como múltiples bifurcaciones, una especie de red interconectada por focos nodales que son modificados de acuerdo con las coyunturas hegemónicas e históricas, funciona a través de la expansión de mecanismos de interacción. Por supuesto, la globalización y la utilización de este término, trasciende el periodo de finales del siglo XX e inicios del siglo $\mathrm{XXI}$, no obstante, este trabajo se enfoca en los cambios que produce en la coyuntura de las políticas neoliberales. En la siguiente fase se argumenta cómo, a pesar de las fuerzas localizadas en relación con la globalización, existen escenarios de posibilidades para buscar la transformación en los procesos educativos.

\section{Conclusiones}

Como se ha expuesto a lo largo de este ensayo, el proceso de globalización en la etapa neoliberal se ha convertido en el medio para implementar cambios estructurales en los entornos sociales, culturales, políticos y económicos. Este escenario muestra las estrategias utilizadas históricamente por los grupos hegemónicos de poder. Ellos han controlado el acceso, la construcción de conocimiento y la vez, marcado estas dinámicas como espacios de disparidad en donde la colonialidad se mantiene presente.

De la misma manera, la gramática de este proceso involucra que exista más de un cúmulo de intencionalidades, por lo cual, las posibilidades para beneficiarse de los flujos propuestos por la globalización están presentes y, lejos de reproducir discursos estandarizados, se 
pueden vislumbrar una serie de caminos para pensar en transformar los ambientes educativos. Esa es una de las metas marcadas por nuestros argumentos, la posibilidad de generar espacios para poder trabajar con consciencia crítica a pesar de las demandas que provienen de un contexto cada vez más exigente y de un mercado que requiere la reproducción acrítica de una serie de saberes.

La incidencia contextual de los procesos genera preocupación constante por las funciones del profesorado. Un cuerpo docente de educación superior comprometido, en la mayoría de los casos, con la preparación de futuros profesionales para cumplir funciones que exigen compromiso, visión, disposición al trabajo colaborativo y reconocimiento de las dinámicas de este contexto cambiante, cuando muchas de las constantes actuales se encuentran marcadas por la competencia, individualismo y la necesidad de cuidar a toda costa los puestos de trabajo.

Esto genera incertidumbre en el profesorado. Este personal observa cómo se recortan espacios para el trabajo dentro y fuera de las aulas. Además, se disminuyen partidas para poder realizar procesos de investigación y extensión, propios de la función social universitaria, o cuando menos, las condiciones se precarizan, pues se deben arañar fondos o, por el contrario, estos mismos no existen. Asimismo, la inestabilidad se refleja en la multiplicidad de funciones de índole instrumental que reducen el tiempo para la praxis educativa y la convierten en una educación para el silencio y de corte bancario (Streck et al., 2015).

Los hallazgos encontrados a partir del análisis de esta producción posibilitan cuestionar los procesos relacionados con la construcción del conocimiento, la preparación del mismo currículo, el juego dicotómico entre la teoría y la práctica, la preparación del profesorado para la construcción de lo deseable, en términos esperanzadores. Se parte del debate sobre la transformación educativa, de un presente con visión crítica que reconozca las fuerzas que inciden sobre las concepciones de la realidad. Esto, con el objetivo de profundizar en la beta de conciencia crítica que posibilitan los espacios marcados por la innovación educativa y las posibilidades de un entramado global.

No obstante, no puede dejarse de lado la constante reflexión y socialización que debe existir sobre las estrategias de control y las formas en las cuales la ciudadanía es parte de maniobras operadas con mucha pericia, para mantener las desigualdades existentes entre quienes tienen el conocimiento necesario y quienes poseen saberes que poco a poco 
son dejados de lado debido a las necesidades/intereses de un mercado que valora a unos seres sobre otros. Estos son los desafíos que el profesorado comprometido debe afrontar de manera conjunta y no por medio de espacios separados.

Sobre dicha situación, se considera, como un elemento esencial la apertura de espacios para la preparación de profesorado, además, la revisión del perfil de las personas encargadas en los procesos educativos. Para construir estas modificaciones es pertinente realizar intervenciones encaminadas desde la planificación consciente a largo plazo, comprometida con la transformación de los escenarios educativos y la toma de consciencia de todas las personas involucradas, cuya respuesta se encuentre en la mejora de las condiciones a nivel social.

A partir de lo expuesto, existe la inquietud por generar el reconocimiento contextual y crítico en el estudiantado sobre la incidencia del empuje económico-financiero en relación con la educación y la impronta por rentabilizar cada esfera de acción relacionada con estas, desde el plano de las ganancias económicas o preparación de capital humano que sirva como mano de obra para desempeñar las funciones de la estructura a nivel mundial (Sánchez y Rodríguez, 2011).

Por ende, se propone el trabajo de la mano con la construcción de capacidades y habilidades para reconocer los contextos en los que se desempeñan las funciones y donde toma sentido, la indagación desde múltiples aristas para poder comprender el trasfondo semántico de algunas propuestas o reformas educativas y las consecuencias que las mismas puedan producir en el tejido social. Estos procesos de avance vertiginoso tecnológico pueden convertirse en una herramienta para el proceso cognitivo de la población estudiantil y la transformación en la forma como se construyen los procesos de enseñanza y aprendizaje.

Por esos motivos, se reitera la importancia de tomar en cuenta cómo, en medio de un contexto cambiante, la sociedad costarricense afronta un sinnúmero de retos cuando el impacto de la globalización incide en las relaciones sociales existentes dentro y fuera de las instituciones educativas. Este escenario a la vez presenta desafíos para los involucrados y comprometidos con el proceso de enseñanza y aprendizaje. Además, modifica el tejido social, así como las competencias de las personas para encarar las transformaciones presentadas por el entorno. En ese sentido, se contraen compromisos con la posibilidad de 
aprender y desarrollar destrezas que permitan insertarse dentro de las dinámicas propuestas por el variable contexto.

Como se ha mencionado, desde la presente perspectiva, algunas de los elementos particulares que deberían estar presentes en esta comunión expresada, van de la mano con la visualización de modelos de educación contextualizados, de la toma de consciencia crítica sobre las dinámicas presentes en la toma de decisiones políticas, económicas, sociales, culturales. No obstante, existe dificultad para realizar estos procesos de forma individual, por lo cual, las redes y la socialización se convierten en prácticas significativas para cuestionar las estructuras en las que se desenvuelve la ciudadanía y proponer otros escenarios de acción.

A la luz del ejercicio realizado en las líneas anteriores, se debe reconocer que han quedado muchos temas por debatir o profundizar. Pero como parte de esta dinámica, la puerta queda abierta para seguir discutiendo y dilucidando escenarios desde donde las investigaciones, de un profesorado consciente y participativo esté presente. No obstante, no se olvida el objetivo de búsqueda y aprendizaje de forma continua que permita reconocer que la luz no es incuestionable, sino una forma de comprender los muchos puntos de vista de este caleidoscópico constructo social. En la oscuridad todo es miedo, acostumbrarse a ella deja a la humanidad encerrada ante las posibilidades que surjan, lo mismo que estar muy cerca de la luz puede dejarla ciega.

\section{Referencias}

Apple, M. (2001). Política cultural y educación. Ediciones Morata Blanco Súarez, J., Duarte Romero, L y Aragón Salazar, M. (2017). Neoliberalismo y escuela. Transformaciones de la escuela a partir de las políticas neoliberales en Colombia. Revista Republicana, 23, 93-128. http://dx.doi.org/10.21017/Rev.Repub.2017.v23.a32

Castells, M. (2001). La ciudad de la nueva economía. Papeles de población, 7 (27), 207-221. http://www.redalyc.org/ pdf/112/11202708.pdf

Escárcega, R. (2014). Pedagogía tradicional y pedagogía crítica. Doble Hélice Ediciones-Instituto Latinoamericano de Pedagogía Crítica.

Escuela de Ciencias de la Computación e Informática. (s. f.). Historia del desarrollo de la carrera en la Universidad de Costa Rica. https:// www.ecci.ucr.ac.cr/\%C2\%BFqui\%C3\%A9nes-somos/historia 
Estado de la Nación. (2011). Tercer Informe Estado de la Educación. http://hdl.handle.net/20.500.12337/675

Ferrer, A. (2013). La importancia de las ideas propias sobre el desarrollo y la globalización. Problemas del desarrollo, 173(44), 163174. http://dx.doi.org/10.1016/S0301-7036(13)71879-0

Gurdián, A. (2010). "Al Toro por los cuernos...": Crisis es riesgo y oportunidad. Revista Actualidades Investigativas en Educación 10, 1-15. http://dx.doi.org/10.15517/aie.v10i4.10158

Huerta, M. (2005). El neoliberalismo y la conformación del Estado subsidiario. Política y Cultura, 24, 121-150. http://www.scielo.org.mx/ scielo.php?script=sci_arttext\&pid=S0188-77422005000200006

Lander, E. (Comp.) (2000). La colonialidad del saber, eurocentrismo y ciencias sociales. Perspectivas latinoamericanas. CLACSO.

Núñez, B. (1974). Hacia la universidad necesaria. https://documentos. una.ac.cr/bitstream/handle/unadocs/5972/hacia\%20la\%20Universidad $\% 20$ Necesaria.pdf? sequence $=1 \&$ isAllowed $=y$

Pascual, R y Ghiotto, L. (2010). Reconceptualizando lo político: Estado, mercado mundial, globalización y neoliberalismo. Nueva Época, 23(64), 133-162. http://www.scielo.org.mx/scielo. php?script=sci_arttext\&pid=S0187-57952010000300006

Rama, C. (2017). La evolución de la educación superior. En M. Román, V. Lentini y Y. Thyme (Eds.), La reforma de la calidad: Estructuras, dinámicas y debilidades del aseguramiento de la calidad de la educación superior en América Latina y el Caribe (pp. 1-69). Programa Estado de la Nación.

Sánchez, P. y Rodríguez, J. (2011). Globalización y educación: Repercusiones el fenómeno en los estudiantes y alternativas frente al mismo. Revista Iberoamericana de Educación, 54,1-12. https:// doi.org/10.35362/rie5451653

Sánchez, J. y Zaldívar, M. (2015). La responsabilidad social como respuesta a los efectos de la globalización y el neoliberalismo. Revista ECA Sinergia, 7(2), 142-149. http://oaji.net/articles/2017/5813-1516137834.pdf

Solano, J. (2018). Las narrativas del desarrollo en América Latina y la nueva gramática social del capitalismo tardío. Letra Maya.

Streck, R., Redin, E, y Zitkoski, J. (2015). Diccionario Paulo Freire. CEAAL. Torres, J. (2001). Educación en tiempos de neoliberalismo. Editorial Morata. 
